\title{
Rotation of the Mass Donors in High-mass X-ray Binaries and Symbiotic Stars
}

\author{
K. Stoyanov ${ }^{1}$, R. Zamanov ${ }^{1}$ \\ ${ }^{1}$ Institute of Astronomy and National Astronomical Observatory, Bulgarian Academy of Sciences, 72 Tsarigradsko \\ Shousse Blvd., 1784 Sofia, Bulgaria
}

Corresponding author: kstoyanov@astro.bas.bg

\begin{abstract}
Our aim is to investigate the tidal interaction in High-mass X-ray Binaries and Symbiotic stars in order to determine in which objects the rotation of the mass donors is synchronized or pseudosynchronized with the orbital motion of the compact companion. We find that the Be/X-ray binaries are not synchronized and the orbital periods of the systems are greater than the rotational periods of the mass donors. The giant and supergiant High-mass X-ray binaries and symbiotic stars are close to synchronization. We compare the rotation of mass donors in symbiotics with the projected rotational velocities of field giants and find that the $\mathrm{M}$ giants in S-type symbiotics rotate on average 1.5 times faster than the field $\mathrm{M}$ giants. We find that the projected rotational velocity of the red giant in symbiotic star MWC 560 is $v \sin i=8.2 \pm 1.5 \mathrm{~km} \mathrm{~s}^{-1}$, and estimate its rotational period to be $P_{\text {rot }}=144-306$ days. Using the theoretical predictions of tidal interaction and pseudosynchronization, we estimate the orbital eccentricity $e=0.68-0.82$.
\end{abstract}

Keywords: stars: binaries: symbiotic - stars: rotation - stars: late type - stars: binaries: close - X-rays: binaries.

\section{Introduction}

A High-mass X-ray binary system consists of a compact object (a neutron star or a black hole) accreting material from an $\mathrm{O}$ or $\mathrm{B}$ companion star. They are divided into $\mathrm{Be} / \mathrm{X}$-ray binaries (main-sequence star as a companion) and Giant/Supergiant X-ray binaries (giant or supergiant star as a companion). Accretion of matter is different for both types of X-ray binaries. In the Be/X-ray binaries, the compact object crosses the circumstellar disc and accretes matter from that disk. In the giant/supergiant X-ray binaries, the mass donor ejects a slow and dense wind radially outflowing from the equator and the compact object directly accretes the stellar wind through Bondi-Hoyle-Lyttleton accretion.

Symbiotic stars are interacting binaries, consisting of an evolved giant (either a normal red giant in S-types symbiotics or a Mira-type variable in D-types symbiotics) transferring mass to a hot and luminous white dwarf or neutron star. The symbiotic stars are surrounded by a rich and luminous nebula resulting from the presence of both an evolved giant with a heavy mass-loss and of a hot companion abundant in ionizing photons and often emanating its own wind.

\section{Synchronization and Pseudosynchronization}

In a binary with a circular orbit the rotational period of the primary, $\mathrm{P}_{\text {rot }}$, reaches an equilibrium value at the orbital period, $P_{\text {orb }}=P_{\text {rot }}$. In other words the synchronous rotation (synchronization) means that the rotational period is equal to the orbital period. In a binary with an eccentric orbit, the corresponding equilibrium is reached at a value of $P_{\text {rot }}$ which is less than $P_{\text {orb }}$, the amount less being a function solely of the orbital eccentricity $e$. In practice, in a binary with an eccentric orbit the tidal force acts to synchronize the rotation of the mass donor with the motion of the compact object at the periastron - pseudosynchronous rotation. To calculate the period of pseudosynchronization, $\mathrm{P}_{p s}$, we use (Hut 1981):

$$
P_{p s}=\frac{\left(1+3 e^{2}+\frac{3}{8} e^{4}\right)\left(1-e^{2}\right)^{\frac{3}{2}}}{1+\frac{15}{2} e^{2}+\frac{45}{8} e^{4}+\frac{5}{16} e^{6}} P_{o r b} .
$$

\subsection{Stars with radiative envelopes}

Following Hurley, Tout \& Pols (2002) the circularization timescale for stars with radiative envelopes is:

$$
\frac{1}{\tau_{\text {circ }}}=\frac{21}{2}\left(\frac{G M_{1}}{R_{1}^{3}}\right)^{\frac{1}{2}} q_{2}\left(1+q_{2}\right)^{\frac{11}{6}} E_{2}\left(\frac{R_{1}}{a}\right)^{\frac{21}{2}},
$$


where $\mathrm{M}_{1}$ and $\mathrm{R}_{1}$ are the mass and the radius of the primary respectively, $\mathrm{q}_{2}$ is the mass ratio $\mathrm{M}_{2} / \mathrm{M}_{1}$, and $a$ is the semi-major axis. The second-order tidal coefficient $\mathrm{E}_{2}=1.592 \times 10^{-9} M_{1}^{2.84}$.

The synchronization time scale is given as,

$$
\tau_{\text {sync }}=K \tau_{\text {circ }},
$$

where $\mathrm{K}$ is:

$$
K \approx \frac{0.015}{r_{g}} \frac{1+q_{2}}{q_{2}}\left(\frac{R_{1}}{a}\right)^{2} .
$$

For the gyration radius of the primary $r_{g}$ we adopt $r_{g} \approx 0.16$ for giants, and $r_{g} \approx 0.25$ for main sequence stars (Claret \& Gimenez, 1989).

\subsection{Stars with convective envelopes}

Following Hurley, Tout \& Pols (2002) the synchronization timescale for stars with convective envelopes is:

$$
\tau_{\text {syn }} \approx 800\left(\frac{M_{1} R_{1}}{L_{1}}\right)^{1 / 3} \frac{M_{1}^{2}\left(\frac{M_{1}}{M_{2}}+1\right)^{2}}{R_{1}^{6}} P_{\text {orb }}^{4} \quad \text { yr },
$$

where $L_{1}$ is the luminosity of the giant.

The circularization time scale is:

$$
\frac{1}{\tau_{\text {circ }}}=\frac{21}{2}\left(\frac{k}{T}\right) q_{2}\left(1+q_{2}\right)\left(\frac{R_{1}}{a}\right)^{8} .
$$

In Eq. $6,(k / T)$ is:

$$
\left(\frac{k}{T}\right)=\frac{2}{21} \frac{f_{\text {conv }}}{\tau_{\text {conv }}} \frac{M_{\text {env }}}{M_{1}} \mathrm{yr}^{-1},
$$

where $R_{\mathrm{env}}$ is the depth of the convective envelope, $M_{\text {env }}$ is the envelope's mass, and

$$
\tau_{\text {conv }}=0.4311\left(\frac{M_{\text {env }} R_{\text {env }}\left(R_{1}-\frac{1}{2} R_{\text {env }}\right)}{3 L_{1}}\right)^{\frac{1}{3}} \mathrm{yr}
$$

is the eddy turnover time scale (the time scale on which the largest convective cells turnover). The numerical factor $f_{\text {conv }}$ is

$$
f_{\text {conv }}=\min \left[1,\left(\frac{P_{\text {tid }}}{2 \tau_{\text {conv }}}\right)^{2}\right],
$$

where $P_{\text {tid }}$ is the tidal pumping time scale given by

$$
\frac{1}{P_{\text {tid }}}=\left|\frac{1}{P_{\text {orb }}}-\frac{1}{P_{\text {rot }}}\right| .
$$

The pseudosynchronization timescale is $\tau_{\mathrm{ps}}=$ $(7 / 3(\alpha-3)) \tau_{\text {circ }}$, where $\alpha$ is a dimensionless quantity, representing the ratio of the orbital and rotational angular momentum:

$$
\alpha=\frac{q_{2}}{1+q_{2}} \frac{1}{r_{\mathrm{g}}^{2}}\left(\frac{a}{R_{1}}\right)^{2} .
$$

For a red giant we adopt $r_{\mathrm{g}} \approx 0.3$ (Claret, 2007).

In all equations, the masses, the radii and the lumunosities are in solar units.

\section{High-mass X-ray Binaries}

The orbital and stellar parameters of 13 High-mass Xray binaries are given in Table 1 and Table 2 in Stoyanov \& Zamanov (2009). We add 2 more objects $4 \mathrm{U} 2206+54$ and MWC 148. The orbital and stellar parameters are taken from Ribó et. al. (2006) and Casares et al. (2012) respectively.

Using Eq. 2 and Eq.3 we estimate the circularization and synchronization timescales. The results are given in Table 1. The lifetime of a star on the main sequence can be estimated as $\tau_{M S}=10^{10}\left(M_{\odot} / M\right)^{2.5}$ years (Hansen \& Kawaler, 1994). Comparing these lifetimes with $\tau_{\text {sync }}$ from Table 1, we see that among the Be/X-ray binaries only for LSI $+61^{0} 303$ is $\tau_{\text {sync }} \sim \tau_{M S}$. This is the only $\mathrm{Be} / \mathrm{X}$-ray binary for which we can expect considerable changes of the rotation of the primary during the lifetime of the Be star.

The lifetime of the giant is comparable or longer then $\tau_{\text {circ }}$ and $\tau_{\text {sync }}$ for the giant/supergiant systems with short orbital periods. The exceptions are V725 Tau and BP Cru, for which $\tau_{\text {sync }}$ and $\tau_{\text {circ }}$ are longer than the lifetime of the giant/supergiant stage.

On Fig.1 in Stoyanov \& Zamanov (2009) is plotted $\mathrm{P}_{r o t}$ versus $\mathrm{P}_{p s}$. The giant/supergiant systems are located close to the line $\mathrm{P}_{p s}=P_{r o t}$, while those with mass donors from spectral class $\mathrm{V}$ are far away from the equilibrium.

In the Be/X-ray systems BQ Cam, V635 Cas, V725 Tau and $4 \mathrm{U} 2206+54$, the tidal force spinning down the donor star. For the system LSI+6 $61^{0} 303$, the rotation of the mass donor is close to pseudosynchronization. This is the only $\mathrm{Be} / \mathrm{X}$-ray binary in which $\tau_{\text {sync }}$ is comparable with the life-time of the binary. In the binaries X Per and MWC 148, the neutron star is far away from the Be star and the tidal force is weak.

Giant and supergiant systems are close to (pseudo)synchronization. In these binaries the rotation of the mass donors is influenced by the presence of the compact object. In LMC X-4 and Cen X-3, the mass donors are synchronized and the orbits are circularized. With respect to the rotation of the mass donor, V725 Tau is similar to the Be/X-ray binaries. Cyg X-1 is synchronized and almost circularized. V830 Cen is pseudosynchronized but not circularized yet. The systems LSI $+65^{0} 010$ and Vela X-1 are close to pseudosynchronization and the tidal force accelerates the rotation of the mass donors. In the case of SMC X-1, the tidal force acts as a decelerator of the rotation of the mass donor. In $\mathrm{BP} \mathrm{Cru}$, a gas stream from the mass donor 
exists, probably resulting from the strong tidal force and spin-up of the mass donor (Leahy \& Kostka, 2008).

Table 1: Calculated time scales. Given here are the name of the object, synchronization time scale, circularization time scale, and the lifetime.

\begin{tabular}{lclc}
\hline object & $\tau_{\text {sync }}[\mathrm{yr}]$ & $\tau_{\text {circ }}[\mathrm{yr}]$ & lifetime $[\mathrm{yr}]$ \\
\hline Be/X-ray binaries & & \\
LSI+610 $303^{*}$ & $2.8 \times 10^{7}$ & $2.4 \times 10^{8}$ & $5.6 \times 10^{7}$ \\
X Per & $6.2 \times 10^{17}$ & $1.8 \times 10^{21}$ & $1.1 \times 10^{7}$ \\
BQ Cam & $3.5 \times 10^{11}$ & $7.6 \times 10^{13}$ & $3.9 \times 10^{6}$ \\
V635 Cas & $1.4 \times 10^{11}$ & $9.5 \times 10^{12}$ & $7.3 \times 10^{6}$ \\
4U 2206+54 & $4.9 \times 10^{9}$ & $3.7 \times 10^{11}$ & $7.3 \times 10^{7}$ \\
MWC 148 & $1.2 \times 10^{17}$ & $5.1 \times 10^{20}$ & $9.8 \times 10^{7}$ \\
Giant systems & & \\
V725 Tau & $2.8 \times 10^{12}$ & $8.0 \times 10^{14}$ & $4 \times 10^{5}$ \\
LMC X-4 & $4.5 \times 10^{2}$ & $7.7 \times 10^{2}$ & $1 \times 10^{6}$ \\
Cen X-3 & $2.3 \times 10^{3}$ & $4.2 \times 10^{3}$ & $5 \times 10^{5}$ \\
Supergiant systems & & \\
V830 Cen & $7.5 \times 10^{3}$ & $1.4 \times 10^{4}$ & $1 \times 10^{6}$ \\
LSI+65 010 & $1.3 \times 10^{4}$ & $3.9 \times 10^{4}$ & $1 \times 10^{6}$ \\
Vela X-1 & $1.0 \times 10^{4}$ & $2.8 \times 10^{4}$ & $3.9 \times 10^{5}$ \\
SMC X-1 & $3.3 \times 10^{4}$ & $8.2 \times 10^{4}$ & $8.8 \times 10^{5}$ \\
BP Cru & $1.8 \times 10^{6}$ & $8.8 \times 10^{6}$ & $8 \times 10^{4}$ \\
Cyg X-1 & $<1$ & $<1$ & $1 \times 10^{5}$ \\
* & $<$ aswing neutron star as a secondary component.
\end{tabular}

*assuming neutron star as a secondary component.

\section{S-type Symbiotic Stars}

43 symbiotic stars have been observed with FEROS spectrograph at the $2.2 \mathrm{~m}$ ESO telescope of the La Silla Observatory (Zamanov et al. 2007). The data for the rotation of 55 field red giants are taken from the literature. $\mathrm{M}$ giants in S-type symbiotics rotate faster than the field $\mathrm{M}$ giants. Histograms of the available $v \sin i$ data for the red giants are plotted in Fig.2 in Zamanov \& Stoyanov (2012). For the field M0III-M6III giants we calculate a mean $v \sin i=5.0 \mathrm{~km} \mathrm{~s}^{-1}$, median $v \sin i=4.3 \mathrm{~km} \mathrm{~s}^{-1}$, and standard deviation of the mean $\sigma=4.0 \mathrm{~km} \mathrm{~s}^{-1}$. For the M0III-M6III giants in symbiotics, we get a mean $v \sin i=7.8 \mathrm{~km} \mathrm{~s}^{-1}$, median $v \sin i=8.0 \mathrm{~km} \mathrm{~s}^{-1}$, and standard deviation of the mean $\sigma=2.1 \mathrm{~km} \mathrm{~s}^{-1}$.

There are 5 objects in our sample that deviate from the synchronization. These objects are RS Oph, MWC 560, CH Cyg, CD-43 ${ }^{\circ} 14304$ and Z And. In three of them collimated jets are detected: Z And (Skopal et al. 2009); CH Cyg (Crocker et al. 2002), MWC 560
(Tomov et al. 1990). Additionally to the jets, ejection of blobs are detected from RS Oph and $\mathrm{CH}$ Cyg (Iijima et al. 1994). This confirms the suggestions that in the jet-ejecting symbiotics the mass donors rotate faster than the orbital periods. Probably there is a link between the jets and the mass donor rotation.

On Fig. 1 are plotted together the High-mass X-ray binaries and the S-type symbiotic stars. It shows that none of the objects in our sample is above the line of synchronization.

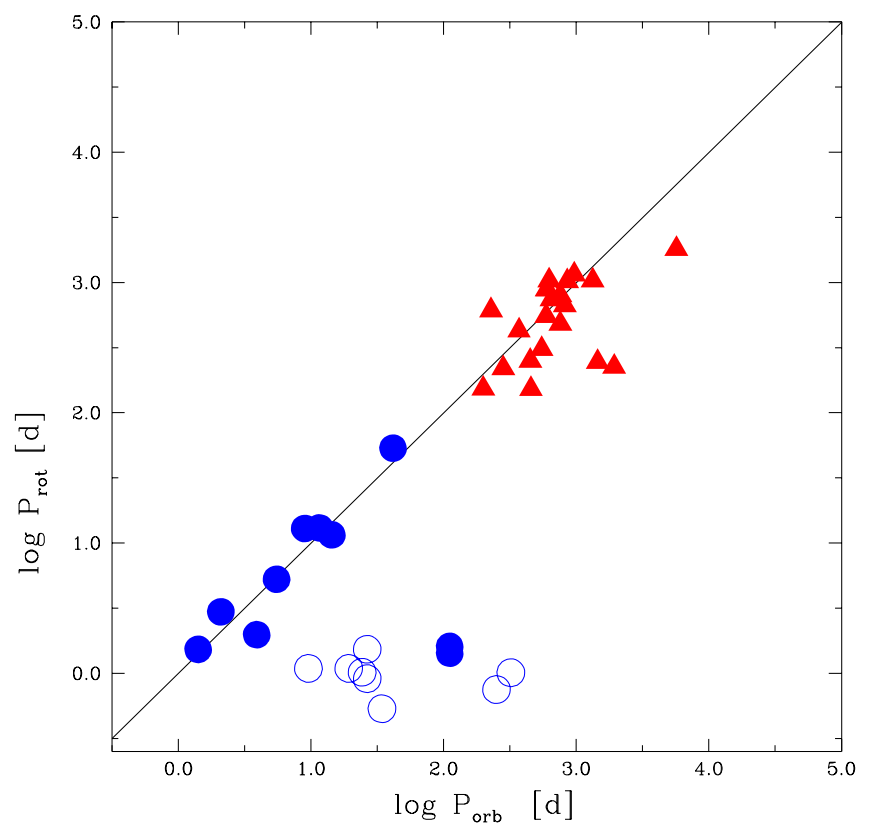

Figure 1: $\mathrm{P}_{\text {rot }}$ versus $\mathrm{P}_{\text {orb }}$ on a logarithmic scale. The blue circles indicates the High-mass X-ray Binaries filled symbols for giant/supergiant systems and open symbols for Be/X-ray binaries. The red triangles indicates the S-type symbiotic stars.

\section{Orbital Eccentricity of MWC 560}

MWC 560 is a symbiotic star, which consists of a red giant and a white dwarf (Tomov et al. 1990). The most spectacular features of this object are the collimated ejections of matter with velocities of up to $\sim 6000 \mathrm{~km} \mathrm{~s}^{-1}$ (Tomov et al. 1992) and the resemblance of its emission line spectrum to that of the low-redshift quasars (Zamanov \& Marziani, 2002). The jet ejections are along the line of sight and the system is seen almost pole-on $\left(i<16^{\circ}\right)$. This makes it difficult to obtain the orbital eccentricity of the system in a conventional way.

For the system we adopt $R_{\mathrm{g}}=140 \pm 7 R_{\odot}, L_{\mathrm{g}} \sim$ $2400 L_{\odot}, M_{\mathrm{g}}=1.7 M_{\odot}, M_{\mathrm{wd}}=0.65 M_{\odot}, P_{\text {orb }}=$ $1931 \pm 162$ day (Gromadzki et al. 2007), $R_{\text {env }}=0.9 R_{\mathrm{g}}$ 
Rotation of the Mass Donors in High-mass X-ray Binaries and Symbiotic Stars

and $M_{\text {env }}=1.0 M_{\odot}$ (Herwig 2005). With the above values of the parameters assumed, we derive the semimajor axis of the orbit to be $a \approx 860 R_{\odot}$. Using these parameters, we calculate from Eq. 5 and Eq. 6 the synchronization and circularization time scales: $\tau_{\text {sync }}=$ $2.6 \times 10^{4} \mathrm{yr}$ and $\tau_{\text {circ }}=3.1 \times 10^{6} \mathrm{yr}$. The typical lifetime of a symbiotic star is $\tau_{\mathrm{ss}} \sim 10^{5} \mathrm{yr}$ (Yungelson et al., 1995). From the rate of accretion on the white dwarf, $\dot{M}_{\text {acc }} \approx 5 \times 10^{-7} M_{\odot}$ (Schmid et al. 2001), we can estimate, that it will take $10^{6} \mathrm{yr}$ to accrete $\sim 0.5 M_{\odot}$ from the envelope of the red giant companion. Because the giant also losses mass via stellar wind, we find that the lifetime of the symbiotic phase of MWC 560 should be $\tau_{\mathrm{ss}} \leq 10^{6}$ yr. For MWC 560 we have therefore the situation in which $\tau_{\mathrm{ps}}<\tau_{\mathrm{syn}}<\tau_{\mathrm{ss}}<\tau_{\text {circ }}$. This means that the symbiotic phase is long enough that the tidal forces can (pseudo)synchronize the rotation of the red giant. On the other hand, the value of $\tau_{\text {circ }}$ demonstrates that the symbiotic lifetime of MWC 560 is shorter than the circularization time, and therefore the orbit can be eccentric. This is in agreement with the observational evidences found by Fekel et al. (2007) that the symbiotic stars with $\mathrm{P}_{\text {orb }}>800$ days tend to have eccentric orbits. The above implies that in MWC 560, the red giant is probably synchronized, but the orbit is not circularized. To determine the orbital eccentricity of MWC 560, we need to calculate $P_{\text {rot }}$ for the mass donor. We analyzed 21 high resolution spectra of MWC 560 and obtained value for $v \sin i=8.2 \pm 1.5 \mathrm{~km} \mathrm{~s}^{-1}$. Using $R_{\mathrm{g}}=140 \pm 7 R_{\odot}$ and $i=12^{\circ}-16^{\circ}$, we calculate $P_{\text {rot }}=144-306$ days. This value is less than the orbital period. MWC 560 should be close to synchronization or pseudosynchronization, and $P_{\text {rot }}=P_{\mathrm{ps}}$. Using Eq. 1 we can estimate the orbital eccentricity to be $e=0.68-0.82$.

\section{Conclusions}

Using rotational velocity measurements and the theory of synchronization/pseudosynchronization we:

(1) find that the Be/X-ray binaries are far away from (pseudo)synchronization. The tidal force in the $\mathrm{Be} / \mathrm{X}$-ray binaries acts as a decelerator of the rotation of the mass donors. The only Be/X-ray binary which is close to pseudosynchronization is the $\mathrm{LSI}+61^{0} 303$. The objects containing mass donors of spectral class I and III typically have $P_{\text {rot }} \sim P_{p s}$ and are close to (pseudo)synchronization;

(2) demonstrate that the $\mathrm{M}$ giants in symbiotic stars rotate faster than the field giants. Most symbiotics with orbital period less than $1000 \mathrm{~d}$ are synchronized;

(3) show that the High-mass X-ray binaries and the S-type symbiotic stars are either on the line of synchronization or they are under the line. None of the objects in our sample is above the line of synchronization.
(4) calculate that the orbit of the symbiotic star MWC 560 should be highly eccentric, with $e \sim 0.7$.

\section{Acknowledgement}

We thank the anonymous referee for constructive comments. This work was supported by the OP "HRD", ESF and Bulgarian Ministry of Education, Youth and Science under the contract BG051PO001-3.3.06-0047.

\section{References}

[1] Casares, J., Ribó, M., Ribas, I., Paredes, J. M., Vilardell, F., \& Negueruela, I.: 2012, MNRAS, 421, 1103 doi:10.1111/j.1365-2966.2011.20368.x

[2] Claret, A.: 2007, A\&A 467, 1389

[3] Claret, A., \& Gimenez, A.: 1989, A\&AS, 81, 37

[4] Crocker, M. M., Davis, R. J., Spencer, R. E., et al.: 2002, MNRAS, 335, 1100 doi:10.1046/j.1365-8711.2002.05705. $x$

[5] Fekel, F. C., Hinkle, K. H., Joyce, R. R., Wood, P. R., Lebzelter, T.: 2007, AJ 133, 17

[6] Gromadzki, M., Mikołajewska, J., Whitelock, P. A., Marang, F.: 2007, A\&A 463, 703

[7] Hansen, C. J., \& Kawaler, S. D.: 1994, Stellar Interiors. Physical Principles, Structure, and Evolution, Springer-Verlag

[8] Herwig, F.: 2005, ARA\&A 43, 435 doi:10.1146/annurev . astro.43.072103.150600

[9] Hurley, J. R., Tout, C. A., \& Pols, O. R.: 2002, MNRAS, 329, 897 doi:10.1046/j.1365-8711.2002.05038. $\mathrm{x}$

[10] Hut, P.: 1981, A\&A, 99, 126

[11] Iijima, T., Strafella, F., Sabbadin, F., \& Bianchini, A.: 1994, A\&A, 283, 919

[12] Leahy, D. A., \& Kostka, M.: 2008, MNRAS, 384, 747 doi:10.1111/j.1365-2966.2007.12754. $\mathrm{x}$

[13] Ribó, M., Negueruela, I., Blay, P., Torrejón, J. M., \& Reig, P.: 2006, A\&A, 449, 687

[14] Schmid, H. M., Kaufer, A., Camenzind, M., Rivinius, T., Stahl, O., Szeifert, T., Tubbesing, S., Wolf, B.: 2001, A\&A 377, 206

[15] Skopal, A., Pribulla, T., Budaj, J., et al.: 2009, ApJ, 690, 1222 doi:10.1088/0004-637X/690/2/1222

[16] Stoyanov, K. A., \& Zamanov, R. K.: 2009, Astronomische Nachrichten, 330, 727 
[17] Tomov, T., Zamanov, R., Kolev, D., Georgiev, L., Antov, A., Mikolajewski, M., Esipov, V.: 1992, MNRAS 258, 23 doi:10.1093/mnras/258.1.23

[18] Tomov, T., Kolev, D., Zamanov, R., Georgiev, L., Antov, A.: 1990, Nature 346, 637 doi:10.1038/346637a0

[19] Yungelson, L., Livio, M., Tutukov, A., \& Kenyon, S. J.: 1995, ApJ 447, 656 doi:10.1086/175908
[20] Zamanov, R. K., Bode, M. F., Melo, C. H. F., et al.: 2007, MNRAS, 380, 1053 doi:10.1111/j.1365-2966.2007.12150.x

[21] Zamanov, R., Marziani, P.: 2002, ApJL 571, L77 doi:10.1086/341367

[22] Zamanov, R. K., \& Stoyanov, K. A.: 2012, Bulgarian Astronomical Journal, 18, 41 